\title{
Primary Care Providers' Attitudes and Practices Regarding Cancer Screening in Older Adults.
}

\author{
Randa Sifri \\ Thomas Jefferson University \\ Brooke Salzman \\ Thomas Jefferson University \\ Amy Cunningham \\ Thomas Jefferson University \\ Alexis Silverio \\ Thomas Jefferson University \\ Madalene Zale

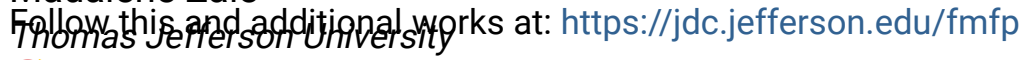 \\ Part of the Family Medicine Commons, Geriatrics Commons, and the Primary Care Commons

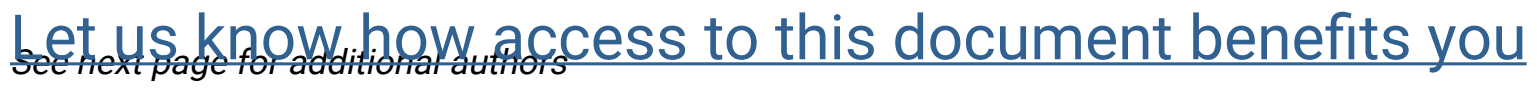

\section{Recommended Citation}

Sifri, Randa; Salzman, Brooke; Cunningham, Amy; Silverio, Alexis; Zale, Madalene; and Talerico, Christine, "Primary Care Providers' Attitudes and Practices Regarding Cancer Screening in Older Adults." (2019). Department of Family \& Community Medicine Faculty Papers. Paper 49.

https://jdc.jefferson.edu/fmfp/49

This Article is brought to you for free and open access by the Jefferson Digital Commons. The Jefferson Digital Commons is a service of Thomas Jefferson University's Center for Teaching and Learning (CTL). The Commons is a showcase for Jefferson books and journals, peer-reviewed scholarly publications, unique historical collections from the University archives, and teaching tools. The Jefferson Digital Commons allows researchers and interested readers anywhere in the world to learn about and keep up to date with Jefferson scholarship. This article has been accepted for inclusion in Department of Family \& Community Medicine Faculty Papers by an authorized administrator of the Jefferson Digital Commons. For more information, please contact: JeffersonDigitalCommons@jefferson.edu. 


\section{Authors}

Randa Sifri, Brooke Salzman, Amy Cunningham, Alexis Silverio, Madalene Zale, and Christine Talerico 
PCP's CRC Attitudes \& Practices in Older Adults

Title: Primary Care Providers' Attitudes and Practices Regarding Cancer Screening in Older Adults

Running title: PCP's CRC Attitudes \& Practices in Older Adults

Author names and affiliations:

Corresponding Author

Randa Sifri, MD

Family and Community Medicine

1015 Walnut St. Suite 401

Philadelphia PA, 19107, United Stated

Randa.sifri@jefferson.edu

$215955-7190$

Brooke Salzman, MD

Family and Community Medicine

1015 Walnut St. Suite 401

Philadelphia PA, 19107, United Stated

Brooke.salzman@jefferson.edu

215 955-6664

Amy Cunningham, PhD, MPH

Family and Community Medicine

1015 Walnut St. Suite 401

Philadelphia PA, 19107, United Stated

Amy.cunningham@jefferson.edu

215 955-0535

Alexis Silverio, MPH, CHES

Family and Community Medicine

1015 Walnut St. Suite 401

Philadelphia PA, 19107, United Stated

Alexis.silverio@jefferson.edu

215-503-6336

Madalene Zale, MPH

Family and Community Medicine

1015 Walnut St. Suite 401

Philadelphia PA, 19107, United Stated

Madalene.zale@jefferson.edu

215 955-1815

Christine Talerico, DO

Family and Community Medicine

1015 Walnut St. Suite 401

Philadelphia PA, 19107, United Stated

cctalerico@gmail.com 
PCP's CRC Attitudes \& Practices in Older Adults

\section{Conflicting and Competing Interests}

The Authors declare that there is no conflict of interest.

\section{Funding Statement}

This research received no specific grant from any funding agency in the public, commercial, or not-for-profit sectors 
PCP's CRC Attitudes \& Practices in Older Adults

\begin{abstract}
Introduction: Cancer-screening decisions for older adults should be individualized. However, conducting such complex shared decisions may be challenging for primary care providers (PCPs). Additionally, there is little information on how PCPs make these decisions. This study consisted of a provider survey and chart review to assess current PCP approaches to breast and colorectal cancer $(\mathrm{CRC})$ screening with patients age $\geq 75$.
\end{abstract}

Methods: PCP survey questions: panel age, comfort with discussion of screening harms and benefits, screening decision-making process, and discussion style. One Hundred charts were chosen from a random sample of male and female patients $\geq 75$ with a recent office visit. Chart reviews assessed whether providers recommended screening for breast and/or colon cancer in patients $\geq 75$, if there was a documented screening discussion, and if screening was completed.

Results: Fifty-one PCPs completed the survey. PCPs varied in the proportions of older adults they recommended for breast and CRC screening. 90.2\% reported feeling very $(43.1 \%)$ or somewhat $(47.1 \%)$ comfortable discussing reasons for/against screening with older patients. Top screening considerations: life expectancy (84.3\%), patient preference (82.4\%), and severity of medical conditions $(70.6 \%)$. Three-quarters $(74.55 \%)$ reported a shared decision-making approach with discussion of harms/ benefits. Of 61 eligible patients, $8(13.1 \%)$ had a documented discussion regarding mammography. Of 58 patients eligible for CRC screening, $7(12.1 \%)$ had a documented discussion.

Discussion: Findings showed inconsistency in PCP approaches to cancer screening in older adults and in discussion documentation. There is ample room for improvement in standardizing approaches and documentation of cancer screening discussions with older patients. 
PCP's CRC Attitudes \& Practices in Older Adults

Introduction

Recommendations for colorectal and breast cancer screening in older adults lack clarity.

Colorectal cancer (CRC) screening guidelines from the U.S. Preventive Services Task Force (USPSTF) state that for people ages 76 to 85 , the decision to screen should be individualized based on patient health, coexisting comorbidities, and prior screening history. For those ages 86 and older, the USPSTF recommends against colorectal cancer screening due to competing causes of mortality that outweigh the potential benefit. New American Cancer Society (ACS) guidelines recommend individualized CRC screening between the ages of 76 and 85 based on a person's preference, life expectancy, overall health and prior screening history and the cessation of CRC screening at the age of $85 .{ }^{1}$ For breast cancer screening, the USPSTF guidelines state that there is currently insufficient evidence to assess the balance of benefits and harms of screening women aged 75 years or older. ${ }^{2,3}$ The AGS (American Geriatric Society) and ACS each state that screening should not be performed in those with a life expectancy less than 10 years. ${ }^{4,5}$

Variations in recommendations reflect uncertainties about cancer screening in older adults. There is limited evidence to guide recommendations in this population due to the lack of older adults in clinical trials about cancer screening. Guidelines propose that decisions about cancer screening with older adults be based on an individualized approach and consider life expectancy, but fail to provide guidance on how to approach these decisions in clinical practice. How to determine the relative benefits and harms of screening an individual patient, how to estimate life expectancy, and how (and when) to have shared-decision making discussions with patients, are all important questions that need to be addressed. Given time pressures faced by primary care providers (PCPs) ${ }^{6,7}$ the process needs not only to be clear and effective, but also efficient and possibly shared with other members of the healthcare team. Currently, there is little information on how PCP's approach these decisions in practice. 
PCP's CRC Attitudes \& Practices in Older Adults

This two-part study aimed to better understand current approaches and practices with cancer screening in older adults in primary care practices. The first part was a survey of PCPs' self-reported breast and colon cancer screening practices with adults age 75 and older, as well as attitudes about cancer screening in older adults and their comfort level with cancer screening cessation discussions. The second part of the study was a chart review to explore whether PCPs are ordering breast and/or CRC cancer screening for adults age 75 and older and whether PCPs are documenting discussions of shareddecision making in electronic medical records (EMR).

\section{Methods}

A literature review found no validated questionnaires regarding cancer screening in older adults. Therefore, the study team developed a survey based on group consensus. The survey of PCPs was administered on-line and allowed participants to remain anonymous. A total of 110 PCPs were invited to participate via email. Surveyed PCPs included attending physicians, fellows, residents, and nurse practitioners from both the Departments of Family and Community Medicine, and General Internal Medicine at Thomas Jefferson University in Philadelphia, PA. The survey included demographic questions, percentage of panel age 75 and older, whether PCPs routinely recommend breast and/or colon cancer screening for patients ages $75-84$ and $\geq 85$, comfort discussing reasons to screen or not screen for cancer, comfort recommending that a patient stop cancer screening, critical factors that determine whether or not to order cancer screening, general approach to shared-decision making about cancer screening, use of tools to assist with shared-decision making, comfort with a trained health educator facilitating shared-decision making, and three case scenarios asking whether the provider would recommend cancer screening in the specific case context. The scenarios were deliberately constructed to have the harms outweigh the benefits (case 1), the benefits outweigh the harms (case 2), and the harms and benefits to be relatively even (case 3 ). The survey allowed for open-ended responses 
PCP's CRC Attitudes \& Practices in Older Adults

when appropriate. The survey responses were analyzed using descriptive statistics for numeric responses and content analysis of written responses.

The second part of the study involved a chart review which examined current primary care practice in regards to breast and colon cancer screening in older adults. One hundred charts were chosen from a random sample of male and female patients 75 and older with an office visit to the institution's family medicine or geriatric medicine practices in the prior year. The sample was generated from practice IDX billing data and from the EMR Allscripts in January 2017. Patients with a history, or active diagnosis, of breast or colon cancer were excluded as were patients who had breast or colon studies performed for diagnostic or surveillance purposes.

Charts were reviewed by two data abstractors using a predefined protocol to assess whether or not providers were recommending screening for breast and/or colon cancer in patients 75 and older. All documents in the chart including progress notes, labs, procedure results, letters, orders, medical history, diagnoses, images, and consultant correspondence from the time the patient turned 75 were reviewed. Patients with a history or active diagnosis of breast or colon cancer were excluded from the final sample, as were patients who had breast or colon studies performed for diagnostic or surveillance purposes. Men were also excluded from the breast cancer screening sample.

Data extracted from the final sample included relevant demographics, including age, sex, race, and ethnicity. The chart review also examined whether or not there was a documented discussion about the decision to continue or discontinue screening. Discussions were categorized as focusing on: life expectancy, risks/benefits, patient preference, and/or comorbidities. Finally, chart abstractors recorded whether the relevant screening test was completed (for breast cancer screening $\rightarrow$ mammography; for CRC screening $\rightarrow$ colonoscopy or fecal immunochemical test (FIT)). Patient demographic, screening 
PCP's CRC Attitudes \& Practices in Older Adults

discussions and ordering information was analyzed using descriptive statistics. The Institutional Review Board of Thomas Jefferson University approved this study.

\section{$\underline{\text { Results }}$}

\section{Providersurvey}

A total of 51 PCPs completed the survey, for a response rate of 46.4 percent. Respondents included 41 attending physicians [family medicine (18), internal medicine (15), geriatric medicine (8)], 1 nurse practitioner, 1 geriatric fellow, and 8 family medicine residents. The response rate was 46.4 percent. Thirty-one (61\%) providers reported that patients who are age 75 and older comprise $0-25 \%$ of their panel, $10(19.6 \%)$ reported that they comprise $26-50 \%$ of their panel, $9(17.6 \%)$ reported that they comprise $51-75 \%$, and $2(3.9 \%)$ reported that they comprise $>75 \%$.

Table 1 shows the percentage of patients for whom PCPs report recommending screening for breast and colon cancer.

[Insert Table 1.]

Of surveyed PCPs, $90.2 \%$ report feeling very $(43.1 \%)$ or somewhat $(47.1 \%)$ comfortable discussing reasons for and against screening for cancer with patients age $>75$, while $9.8 \%$ of PCPs report feeling not very comfortable with these discussions. 86.3\% of PCPs report feeling very (35.3\%) or somewhat (51.0\%) comfortable recommending that a patient stops cancer-screening, while $13.8 \%$ of PCPs report feeling not very or not at all comfortable with these discussions. Of seven respondents who were not very or not at all comfortable recommending that a patient stop cancer screening, one respondent reported feeling that way because "it's beneficial to screen for cancer at all ages" and two respondents because "my patient would be upset to discuss life expectancy." PCPs were asked to rank the most important three factors that determine whether to order cancer screening for someone age 
PCP's CRC Attitudes \& Practices in Older Adults

$>75$. The top three factors were life expectancy (84.3\%), patient preference (82.4\%), and severity of medical conditions (70.6\%). When presented with different descriptions of current practice in regards to cancer screening with older adults, 38 PCP's (74.5\%) selected that they "present information about the benefits and harms of screening, discuss the pros and cons with the patient, elicit the patient's understanding and preferences, and come to a shared decision." Two PCPs (3.9\%) selected that they "make the decision about who is appropriate to screen and offer screening only to those who they think would benefit"; seven PCPs (13.7\%) selected that they "present information about the benefits and harms of screening to the patient and let him/her decide whether to screen" and three PCPs selected "other."

PCPs were presented with three case scenarios about cancer screening in older patients and asked whether the harms outweigh the benefits, benefits outweigh the harms, harms and benefits are most likely even, or if they were unsure. Forty-two (82.4\%) respondents correctly assessed the scenario in which the harms outweighing the benefits; 29 (56.9\%) chose the correct response for the benefits outweighing harms, and 17 (33.3\%) for the scenario with benefits and harms being even.

When asked if there are tools that they currently use in practice to assist with decision-making about cancer-screening in older adults, 78.4\% of PCPs answered, "No." PCPs reported that they would find the following tools helpful in assisting with decision-making about cancer-screening in older patients: Methods for estimating life expectancy (60.8\%), Decision aids for providers to estimate benefits/harms of cancer screening (64.7\%), and decision aids for patients that present personalized benefits/harms of cancer screening (66.7\%). Most (84.3\%) of PCPs reported feeling comfortable with a trained health educator meeting with patients to share information and discuss the benefits and harms of cancer-screening. 15.6\% of PCPs found it "unacceptable" for patients to be mailed information about cancer screening in older adults. 
PCP's CRC Attitudes \& Practices in Older Adults

Chart review

There were 100 ( $n=100)$ charts reviewed. Thirty-nine charts were excluded from the breast cancer screening sample (31 men and 8 women with an active/prior breast cancer diagnosis or history of breast studies for diagnosis or surveillance), leaving a total of $61(n=61)$ charts reviewed. Forty-two charts were excluded from the CRC screening sample, all for history of colon studies for diagnostic or surveillance purposes, leaving a total of $58(n=58)$ charts reviewed. A description of the demographic characteristic of the study sample can be found in Table 2.

[Insert Table 2.]

Cancer screening discussion documentation and adherence to screening at and after the age of 75 can be found in Figures 1 and 2. Of women eligible for breast cancer screening, eight (13.1\%) had a documented discussion regarding whether or not to continue screening; four discussions centered on patient preferences, and four discussions had unspecified content. Of the eight who had a discussion, five (62.5\%) declined a mammogram (although one had a subsequent mammogram), and three (37.5\%) wished to continue screening. Among the 58 patients eligible for CRC cancer screening, seven(12.1\%) had a documented discussion of whether or not to continue screening; five discussions centered around patient preferences, one focused on risks and benefits, and one discussion had unspecified content. Of the seven patients with a documented CRC screening discussion, all declined screening, although one subsequently completed CRC screening [Insert Figure 1.]

[Insert Figure 2.]

\section{Discussion}

Findings from this two-part study suggest that there is uncertainty and inconsistency in primary care practice about how to approach decisions about cancer screening with older adults. Results of this 
PCP's CRC Attitudes \& Practices in Older Adults

PCP survey demonstrated wide variations in self-reported practice surrounding breast and colon cancer screening with older patients. Such variability may arise from guidelines with different, sometimes conflicting, recommendations. Variations in recommendations for breast and/or colon cancer screening in this PCP survey were widest for individuals age $75-84$, which is expected given the lack of sufficient evidence to recommend for or against cancer screening in this cohort of older adults. Variations in selfreported practice were lower in regards to recommending breast and/or colon cancer screening with patients $>85$, as most of the PCP's did not recommend breast and/or colon cancer screening for this group of older adults. This finding may be due to the USPSTF guidelines specifically advising against colon cancer screening in those $>85$, or due to a shared perception of limited benefit of screening for the oldest-old with limited life expectancy.

Self-reports and chart audits are two important ways to evaluate how PCPs approach cancer screening discussions in older adults. Self-reported data can have limits as PCPs could overestimate what they ask in a visit and how often they ask about preventative screenings. Electronic medical record chart audits may provide a clearer picture as to what is actually being done by the PCP during a visit. ${ }^{8}$ In this chart review, the majority of women age 75 and older had a mammogram ordered and yet, only a minority of charts showed any documentation of a discussion about shared-decision making or considerations of whether or not to screen. Only a minority of patients age 75 and older had colon cancer screening ordered. Still, an even smaller percentage of charts showed any documentation of a discussion about shared-decision making or considerations of whether or not to screen. This lack of documentation is concerning, regardless of whether patients are being screened or not. Even when discussions were document, it remained unclear if patients are being informed of the potential benefits and harms of screening and whether they are active participants in the decision. 
PCP's CRC Attitudes \& Practices in Older Adults

Despite variations in cancer screening guidelines for older adults, most organizations

recommend an individualized approach to considering the relative benefits and harms of screening in the context of a patient's health status and life expectancy. However, the processes of determining the benefits and risks for an individual, as well as for defining health status and estimating life expectancy are not widely understood. There is great uncertainty among PCPs on how to estimate 10 -year survival and how to incorporate prognostic information into these decisions. ${ }^{9}$ PCPs feel they lack key information about risks and benefits of screening and how these change with advancing age and varying health status. Still, it is expected that PCPs present balanced information, guide patients through their options, review personalized risks and benefits, and facilitate a shared, informed decision.$^{10}$

This study showed that while a majority of PCP's reported feeling very or somewhat comfortable discussing reasons for or against cancer screening with older adults, nearly ten percent reported feeling not very comfortable. Additionally, $13.8 \%$ report feeling not very or not at all comfortable recommending that a patient stop screening. As PCP recommendations play a significant role in whether patients get cancer screening, these findings have important implications. Guidelines propose avoiding cancer screening in those with a limited life expectancy; yet, some PCPs are uncomfortable making this recommendation all together. A qualitative study of 28 PCPs also identified reluctance among PCPs to stop preventive care that has a long lag time to achieve benefit despite limited life expectancy. ${ }^{9}$ This study identified several sources of this reluctance or discomfort including uncerta inty in predicting prognosis, difficulty discussing prognosis, and concern about patient reactions. In our study, some PCPs who were not comfortable recommending cancer screening cessation agreed that the discussion about life expectancy would upset their patient.

Nearly $80 \%$ of PCPs reported that they do not use tools in clinical practice to assist with cancer screening discussions with their older patients. The majority reported that they would find tools helpful 
PCP's CRC Attitudes \& Practices in Older Adults

to assist with estimating life expectancy and help providers and/or patients weigh the benefits and harms of screening. Tools that estimate life expectancy are widely available on sites such as eprognosis.org; however, PCPs may not be familiar with them or how to apply them in practice for cancer screening. Frameworks for approaching decisions about cancer screening with older patients have been proposed but have not been widely adopted or assessed in clinical practice. ${ }^{11}$ Decision aids have also been developed for older adults to assist with cancer screening decisions and are currently being evaluated in clinical practice. ${ }^{6,12}$

Studies have shown that decision aids can increase knowledge and assist older women to make an informed choice ${ }^{13}$ without adverse impact on decisional conflict or anxiety (AHRQ, Comparative Effectiveness Review \#145, No. 15-EHC002-Ef, 2014). Using DAs may also provide a way to structure the approach and ensure that patients are adequately informed and involved in the decision. A challenge for PCPs in conducting shared decision making regarding cancer screening with older adults is the time needed to have these nuanced discussions. Whether PCPs are comfortable with someone other than themselves, such as a trained health educator, facilitating these discussions is an important que stion. We found that the vast majority of PCPs report feeling comfortable with a trained health educator meeting with patients to share information and discuss the benefits and harms of cancer-screening.

Our study has several limitations. First, it was conducted with providers and patient charts from one urban academic medical center, so the findings may not be generalizable to all settings. The provider survey was created by the study team, and requires validation in a larger provider sample. Slightly fewer than half of the providers completed the survey, which may have led to selection bias: those who responded may not be representative of the full sample. Additionally, the provider survey information on screening practices is self-reported, which may also lead to bias. Our chart review of a random sample of charts for patients 75 and older helped to address this limitation. However, it is 
PCP's CRC Attitudes \& Practices in Older Adults

important to note that providers vary in their documentation of patient discussions, and some may have had cancer screening conversations with patients that were not documented in the EMR. Finally, our provider survey was anonymous, so we could not link their responses to the charts reviewed.

Our study provides multiple avenues for ongoing research. Future studies should examine the provider views on cancer screening discussions with older adults and their documentation practices with larger, random samples of providers and patient charts. Provider survey responses could also be linked to information from their patients' charts to identify potential discrepancies between reported and actual cancer screening discussions and ordering. Additionally, the patient perspective on cancer screening discussions is critically important; our team has conducted a complimentary study on patient perspectives on these discussions and the use of shared decision-making tools. Ultimately, our work and larger-scale follow-up studies will provide the groundwork for developing optimal models of primary care-based shared decision-making regarding cancer screening in older adults.

\section{Acknowledgements}

We would like to acknowledge Anna Bistline for assistance with the background section and literature review. 
PCP's CRC Attitudes \& Practices in Older Adults

\section{References}

1. American Cancer Society. American Cancer Society Guide line for Colorectal Cancer Screening For people at average risk. https://www.cancer.org/cancer/colon-rectal-cancer/detection-diagnosisstaging/acs-recommendations.html. May 20, 2018. Accessed June 14, 2018.

2. Final Update Summary: Colorectal Cancer: Screening. U.S. Preventive Services Task Force. https://www.uspreventiveservicestaskforce.org/Page/Document/UpdateSummaryFinal/colorectalcancer-screening2. June, 2016. Accessed August 14, 2017.

3. Final Update Summary: Breast Cancer: Screening. U.S. Preventive Services Task Force. https://www.uspreventiveservicestaskforce.org/Page/Document/UpdateSummaryFinal/breastcancer-screening1. September, 2016. Accessed August 14, 2017.

4. Oeffinger KC, Fontham ET, Etxioni R, et al. Breast cancer screening for women at average risk: 2015 guideline update from the American Cancer Society. JAMA. 2015;314(15):1599-1614.

5. AGS Choosing Wisely Workgroup. American Geriatrics Society identifies another five things that healthcare providers and patients should question. J Am Geriatr Soc. 2014;62(5):950-960.

6. Schonberg MA, Kistler CE, Nekhlyudov L, et al. Evaluation of a Mammography Screening Decision Aid for Women Aged 75 and Older: Protocol for a Cluster-randomized Controlled Trial. J clin trials. 2014;4:191.

7. Schoenborn NL, Lee K, Pollack CE, et al. OlderAdults' Views and Communication Preferences About Cancer Screening Cessation. JAMA. 2017;177(8):1121-1128.

8. Montano, DE, Phillips, WR. Cancer Screening by Primary Care Physicians: A Comparison of Rates Obtained from Physician Self-Report, Patient Survey, and Chart Audit. Am J Public Health. 1995;85(6):795-800. 
PCP's CRC Attitudes \& Practices in Older Adults

9. Schoenborn NL, Bowman TL, Cayea D, Boyd C, Feeser S, Pollack CE. Discussing strategies primary care clinicians use when stopping cancer screening in older adults. J Am Geriatr Soc. 2016;64(11):e221-e223.

10. DuBenski LL, Schrager S, McDowell H, Wilke LG, Trentham-Dietz A, Burnside ES. Mammography Screenings: Gaps in Patient's and Physician's Needs for Shared Decision-Making. BreastJ. $2017 ; 23(2): 210-214$.

11. Lee SJ, Leipzig RM, Walter LC. Incorporating lag time to benefit into prevention decisions for older adults. JAMA. 2013 Dec 25;310(24):2609-10.

12. Schonberg MA, Hamel MB, Davis RB, et al. Development and evaluation of a decision aid on mammography screening for women 75 years and older. JAMA. 2014;174(3):417-424.

13. Mathieu E, Barratt A, Davey HM, McGeechan K, Howard K, Houssami N. Informed choice in mammography screening: a randomized trial of a decision aid for 70-year-old women. Arch intern med. 2007;167(19):2039-2046. 